\title{
Low heme oxygenase-1 expression promotes gastric cancer cell apoptosis, inhibits proliferation and invasion, and correlates with increased overall survival in gastric cancer patients
}

\author{
QUAN-GUANG REN ${ }^{1 *}$, SHENG-LI YANG ${ }^{1 *}$, PIN-DONG LI ${ }^{1}$, JIN-RONG XIONG ${ }^{2}$, XIEFAN FANG $^{3}$, \\ JIAN-LI HU ${ }^{1}$, QIU-SHUANG WANG ${ }^{1}$, REN-WANG CHEN ${ }^{1}$, YE-SHAN CHEN ${ }^{1}$, LU WEN ${ }^{1}$ and MIAO PENG ${ }^{1}$ \\ ${ }^{1}$ Cancer Center, Union Hospital, Tongji Medical College, Huazhong University of Science and Technology, \\ Wuhan 430022; ${ }^{2} 457$ th Hospital of PLA, Wuhan, Hubei 430012, P.R. China; ${ }^{3}$ Department of Pediatrics, \\ College of Medicine, University of Florida, Gainesville, FL 32610, USA
}

Received March 16, 2017; Accepted August 11, 2017

DOI: $10.3892 /$ or.2017.5967

\begin{abstract}
Heme oxygenase-1 (HO-1) plays a key role in antioxidation, anti-apoptosis, and anti-proliferation in various types of cancers. However, the relationship between HO-1 expression and gastric cancer development remains largely unknown. In this study, the protein expression of HO-1 in human gastric cancer was measured by immunohistochemistry on paraffin sections of 89 paired gastric carcinoma tissues and adjacent non-cancer tissues. The correlation of HO-1 expression with 5-year overall survival rate was estimated. The effects of decreased HO-1 expression by two strands of small interfered RNAs (siRNAs) on cell apoptosis, proliferation, and invasion of gastric cancer cell lines were examined by flow cytometry, the MTT assay, and the cell migration assay, respectively. High expression of HO-1 was detected in $11.2 \%(10 / 89)$ of gastric carcinoma tissues, compared with $1.1 \%(1 / 89)$ in matched adjacent normal tissues, and correlated with a decreased survival rate in gastric cancer patients. There were no significant correlations between HO-1 expression and clinical characteristics. Downregulation of HO-1 expression using two strands of siRNAs promoted apoptosis and inhibited the proliferation and invasion of two gastric cancer cell lines, SGC7901 and MKN-28 cells. This study demonstrated that HO-1 plays a vital role in the development of gastric cancer and may serve as a therapeutic target of this type of cancer.
\end{abstract}

Correspondence to: Professor Jian-Li Hu, Cancer Center, Union Hospital, Tongji Medical College, Huazhong University of Science and Technology, 1277 Jiefang Avenue, Wuhan 430022, P.R. China E-mail: j15199@126.com

*Contributed equally

Key words: heme oxygenase-1, gastric cancer, apoptosis, proliferation, invasion, overall survival rate

\section{Introduction}

Gastric cancer is one of the most commonly diagnosed cancers worldwide, and it is projected that it ranks fourth in incidence and is the third leading cause of death worldwide (1). Although producing low $\mathrm{pH}$ gastric acid, gastric tissue can still be infected with Helicobacter pylori, leading to gastritis $(2,3)$. Stimulations by certain food, such as spicy and hot temperature food and alcohol, often contribute to tissue inflammation and accumulation of reactive oxygen species (ROS) (4). Chronic inflammation along with altered expression of various genes and abnormal local microenvironment leads to the occurrence of gastric cancer (3). Heme oxygenase-1 (HO-1) is one of the predominant genes to reduce inflammation and oxidation in the body (5). HO-1 regulates the expression of many genes and important molecules, and these have been involved in regulation of cell proliferation, migration, and invasion in many types of cancers, including colon (6), lung (7) and breast cancer (8).

HO-1 and its two isomers, HO-2 and HO-3, have an extremely low expression in the physiological state $(5,9)$. HO-1 plays a vital role in the anti-oxidation and anti-inflammation system and is mainly responsible for suppressing inflammation and removing ROS in the tissue microenvironment (10). In the presence of inflammation, including gastritis, $\mathrm{HO}-1$ expression was upregulated in local tissues (11). HO-1 decomposes heme into $\mathrm{CO}, \mathrm{Fe}^{2+}$, and biliverdin. $\mathrm{CO}$ may decrease inflammation and inhibit apoptosis by regulating the P38-MPAK signaling pathway and increasing the expression of BCL-2 (12). The conversion of $\mathrm{Fe}^{2+}$ to $\mathrm{Fe}^{3+}$ could reduce the accumulation of ROS via Fenton reaction $(13,14)$. Biliverdin has strong antioxidative properties, which could inhibit angiogenesis by suppressing the VEGF and HIF-1 signaling pathways (15). Since HO-1 and its downstream signaling mitigate inflammation and repair oxidative stress injury, we hypothesize that HO-1 may play an important role in the occurrence and development of gastric cancer.

In this study, we examined the expression of HO-1 in gastric cancer tissue compared with the peritumoral tissue by immunochemistry. The correlation of HO-1 expression with 
clinical characteristics and prognosis was evaluated in gastric cancer patients. Moreover, the effects of downregulation of HO-1 by two different strands of siRNAs were studied in gastric cancer cell lines. Our results demonstrated the critical functions of HO-1 in gastric cancer.

\section{Materials and methods}

Patients and tissue specimens. The tissue microarray, containing 89 gastric cancer tissues and matched adjacent normal tissues, was purchased from Shanghai Outdo Biotech (Shanghai, China). Clinical characteristics and survival data were obtained from the 89 patients with gastric cancer. The survival data were obtained between October 2008 and July 2015. The gastric cancer patients for the tissue microarray were all diagnosed by history and pathology. The local ethics committee approved this study, and informed consent was obtained from each patient.

Immunohistochemical assay. Primary antibodies against HO-1 (1:500 dilution; Abnova Corp., Taipei, Taiwan) were used in the present experiment. Immunohistochemical staining was carried out as described in our previous study (16). The immunohistochemistry results were evaluated based on positive cell numbers in the cytoplasmic and nuclear staining. The following score rank system for immunohistochemical staining was used: I, no staining; II, $10 \%$ of cells with nuclear staining and/or weak cytoplasmic staining; III, $10-50 \%$ of cells with nuclear staining and/or distinct cytoplasmic staining; and IV, $>50 \%$ of cells with nuclear staining and/or strong cytoplasmic staining. The scores I and II were considered negative expression, and the scores III and IV were considered as positive expression.

Cell culture and siRNA transfection. The two human gastric cancer cell lines, MKN-28 and SGC7901, and the immortalized normal gastric epithelial cell line, GES-1, were gifts from the Cancer Centre at Sun Yat-Sen University. Commercially available HO-1 siRNAs, the empty vector containing a nonsense RNA sequence, and the RNA transfection kit were obtained from Guangzhou Ribo Bio (Guangzhou, China). All cell lines were cultured in Dulbecco's modified Eagle's medium (DMEM, Life Technologies, Carlsbad, CA, USA) with $10 \%$ fetal calf serum (Gibco, Carlsbad, CA, USA). The cell lines at the harvesting logarithmic growth phase were plated on six-well plates at a density of $2 \times 10^{5}$ and cultured in a $37^{\circ} \mathrm{C}$ humidified incubator with a $5 \% \mathrm{CO}_{2}$ atmosphere. Each cell line was treated with either the nonsense RNA sequence (negative control), HO-1 siRNA-1, or HO-1 siRNA-2. Transfection was carried out with siRNA at a concentration of $100 \mathrm{nM}$ according to the instruction of the kit. The mRNA expression of HO-1 was evaluated by quantitative real-time PCR (qPCR).

MTT assay. MKN-28 and SGC7901 cells at the logarithmically growing phase were seeded at a density of $1 \times 10^{4}$ on 96-well plates and transfected with either nonsense RNA, HO-1 siRNA-1, or HO-1 siRNA-2 after overnight culture. At 24,48 , and $72 \mathrm{~h}$ post-transfection, a mixture of $20 \mu \mathrm{l} \mathrm{MTT}$ and $180 \mu 1$ DMED was added to each well. The cells were
Table I. Correlations of HO-1 protein expression with clinicopathological characteristics in the tissue specimens from gastric cancer patients.

\begin{tabular}{|c|c|c|c|c|c|}
\hline \multirow[b]{2}{*}{$\begin{array}{l}\text { Patient } \\
\text { characteristics }\end{array}$} & \multirow[b]{2}{*}{ No. } & \multicolumn{2}{|c|}{ HO-1 expression } & \multirow[b]{2}{*}{$\chi^{2}$} & \multirow[b]{2}{*}{$\mathrm{P}$-value } \\
\hline & & $\begin{array}{c}\text { Low } \\
(n=79)\end{array}$ & $\begin{array}{l}\text { High } \\
(n=10)\end{array}$ & & \\
\hline Age (years) & & & & 0.664 & 0.497 \\
\hline$\leq 65$ & 55 & 50 & 5 & & \\
\hline$>65$ & 34 & 29 & 5 & & \\
\hline Sex & & & & 0.621 & 0.513 \\
\hline Male & 52 & 45 & 7 & & \\
\hline Female & 37 & 34 & 3 & & \\
\hline Tumor size & & & & 0.348 & 0.503 \\
\hline$\leq 5 \mathrm{~cm}$ & 48 & 44 & 4 & & \\
\hline$>5 \mathrm{~cm}$ & 41 & 35 & 6 & & \\
\hline Pathological grade & & & & 4.357 & 0.052 \\
\hline Grade II & 21 & 16 & 5 & & \\
\hline Grade III & 68 & 63 & 5 & & \\
\hline $\mathrm{T}$ classification & & & & 1.731 & 0.189 \\
\hline $\mathrm{T} 1-\mathrm{T} 2$ & 14 & 11 & 3 & & \\
\hline $\mathrm{T} 3-\mathrm{T} 4$ & 75 & 68 & 7 & & \\
\hline $\mathrm{N}$ classification & & & & 0.321 & 0.736 \\
\hline N0-N1 & 34 & 31 & 3 & & \\
\hline $\mathrm{N} 2-\mathrm{N} 3$ & 55 & 48 & 7 & & \\
\hline M classification & & & & 0.530 & 1.000 \\
\hline M0 & 85 & 75 & 10 & & \\
\hline M1 & 4 & 4 & 0 & & \\
\hline Clinical stage & & & & 0.411 & 0.734 \\
\hline I-II & 35 & 32 & 3 & & \\
\hline III-IV & 54 & 47 & 7 & & \\
\hline
\end{tabular}

HO-1, heme oxygenase 1; TNM, tumor node metastasis.

cultured for another $4 \mathrm{~h}$. The supernatant was removed, and $150 \mu \mathrm{l}$ DMSO was added to each well. The absorbance was measured at $490 \mathrm{~nm}$.

Flow cytometric analysis. MKN-28 and SGC7901 cells at the logarithmically growing phase were seeded at a density of $1.5 \times 10^{5}$ on 6 -well plates and transfected with either nonsense RNA, HO-1 siRNA-1, or HO-1 siRNA-2 after overnight culture. The cells were harvested at $24 \mathrm{~h}$ and evaluated for apoptosis by flow cytometry using propidium iodide (PI) and Annexin V, as previously described (17).

Cell invasion assay. MKN-28 and SGC7901 cells were harvested and seeded at a density of $1 \times 10^{4} / 100 \mu 1$ DMEM in the upper chamber. The lower chamber was loaded with $600 \mu \mathrm{l}$ DMEM with $10 \%$ FBS. Both cell lines were transfected with either nonsense RNA, HO-1 siRNA-1, or HO-1 siRNA-2. After 
A

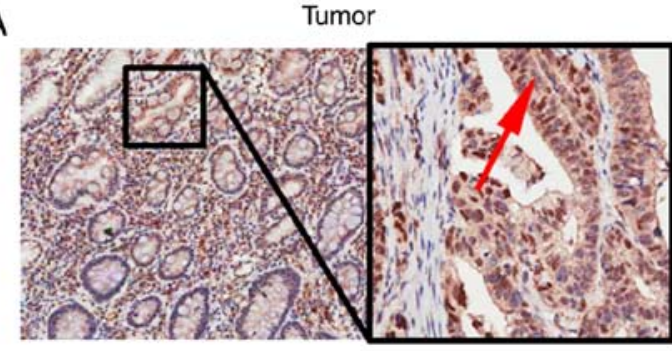

B

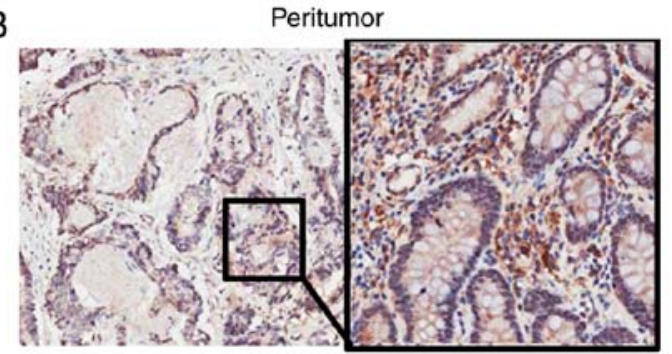

Figure 1. Expression of heme oxygenase-1 (HO-1) in gastric cancer tissues and matched adjacent non-cancer tissues. Representative images show strong HO-1 staining located in the cytoplasm of gastric cancer cells (A) and weak HO-1 staining located in the cytoplasm of the paired peritumoral cells (B).

incubation for $24 \mathrm{~h}$, crystal violet was used to stain the cells in the lower membrane of the chamber. Cells in five randomly selected fields were counted under the microscope.

RNA isolation and reverse transcription PCR. MKN-28 and SGC7901 cells were harvested after being transfected with either nonsense RNA, HO-1 siRNA-1, or HO-1 siRNA-2 for 48 h. TRIzol reagent (Invitrogen, Carlsbad, CA, USA) was used to extract total RNA from cells. These procedures were carried out on ice to prevent RNA degradation. The Nanodrop spectrophotometer (ND-2000; Nanodrop Technology, Wilmington, DE, USA) was used to evaluate the purity and concentration of RNA. The reverse transcription reagent (Takara Bio, Inc., Kusatsu, Shiga, Japan) was used to transcribe RNA to cDNA. The SYBR-Green Prime Script RT-PCR kit (Takara Bio, Inc.) and the Real-time PCR detection system (Applied Biosystems, Foster City, CA, USA) were used to perform qPCR. The internal control was GAPDH, and the primers were: forward, 5'-TCCCATCACCATCTTCCAG-3' and reverse, 5'-GAGCCCCAGCCTTCTCCAT-3'. Three pairs of primers were used to quantitate $\mathrm{HO}-1$ and obtain independent results. The first pair was 5'-GGAGAUUGAGCGCAAC AAG-3' (forward) and 5'-CUUGUUGCGCUAAAUCUCC-3' (reverse). The second pair was 5'-UGAUAGAAGAGGC CAAGAC-3' (forward) and 5'-GUCUUGGCCUCUUCUA UCA-3' (reverse). The third pair was 11 5'-CUGCGUUCCU GCUCAACAU-3' (forward) and 5'-AUGUUGAGCAGGA ACGCAG-3' (reverse). The $2^{-\Delta \Delta C t}$ method was used for fold change calculation.

Western blot analysis. The GES-1, MKN-28, and SGC7901 cells at the logarithmically growing stage were harvested for protein isolation using the protein lysis solution. The protein from each cell line was loaded into $12 \%$ sodium dodecyl sulfate (SDS) polyacrylamide gels and transferred to polyvinylidene difluoride (PVDF) membranes. The membranes were blocked with $5 \%$ milk for $1.5 \mathrm{~h}$, and incubated with a 1:500 dilution of primary antibody against HO-1 (Abnova Corp.) overnight at $4^{\circ} \mathrm{C}$. The primary antibody was visualized with an enhanced chemiluminescence reagent (Beyotime) against the peroxidase-conjugated secondary antibody. The results were analyzed by densitometry analysis. $\beta$-actin was used as an internal control.

Statistical analysis. Statistical analysis was conducted using the SPSS 19.0 software (IBM, Chicago, IL, USA). Each experiment was conducted three times. Experimental results

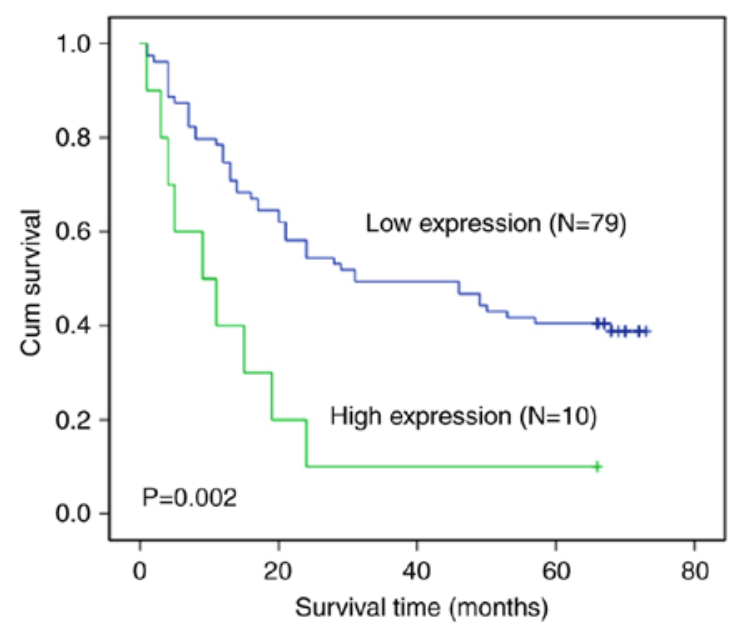

Figure 2. Expression of heme oxygenase-1 (HO-1) in gastric cancer tissues is associated with the overall survival of gastric cancer patients. Low protein expression of HO-1 in gastric cancer tissues correlated with a longer overall survival time in patients compare to that of patients with high protein expression of HO-1 $(\mathrm{P}=0.002)$.

were demonstrated as mean \pm standard deviation (mean \pm SD). Comparisons of clinicopathological parameters between high and low HO-1 expression patients were performed using a two-sided and unpaired t-test. Kaplan-Meier analysis was used to evaluate the survival curves, and the log-rank test was used to analyze prognostic significance. A P-value $<0.05$ was considered to indicate a statistically significant difference.

\section{Results}

HO- 1 expression in gastric cancer tissues and matched adjacent non-cancer tissues. The immunohistochemical assay showed that high expression of HO-1 was present in 10 of 89 (11.2\%) gastric cancer patient tissues. The expression of HO-1 was mainly located in the cytoplasm of gastric cancer cells (Fig. 1A). In contrast, low expression of HO-1 was observed in 1 of $89(1.1 \%)$ matched adjacent non-cancer tissues, with HO-1 expression primarily located in the cytoplasm of adjacent noncancer cells (Fig. 1B).

Correlations between $\mathrm{HO}-1$ expression and clinicopathological characteristics in gastric cancer patients. We next explored the possible correlations between the expression of HO-1 and clinicopathological characteristics in gastric cancer patients. As shown in Table I, there was no statistical signifi- 


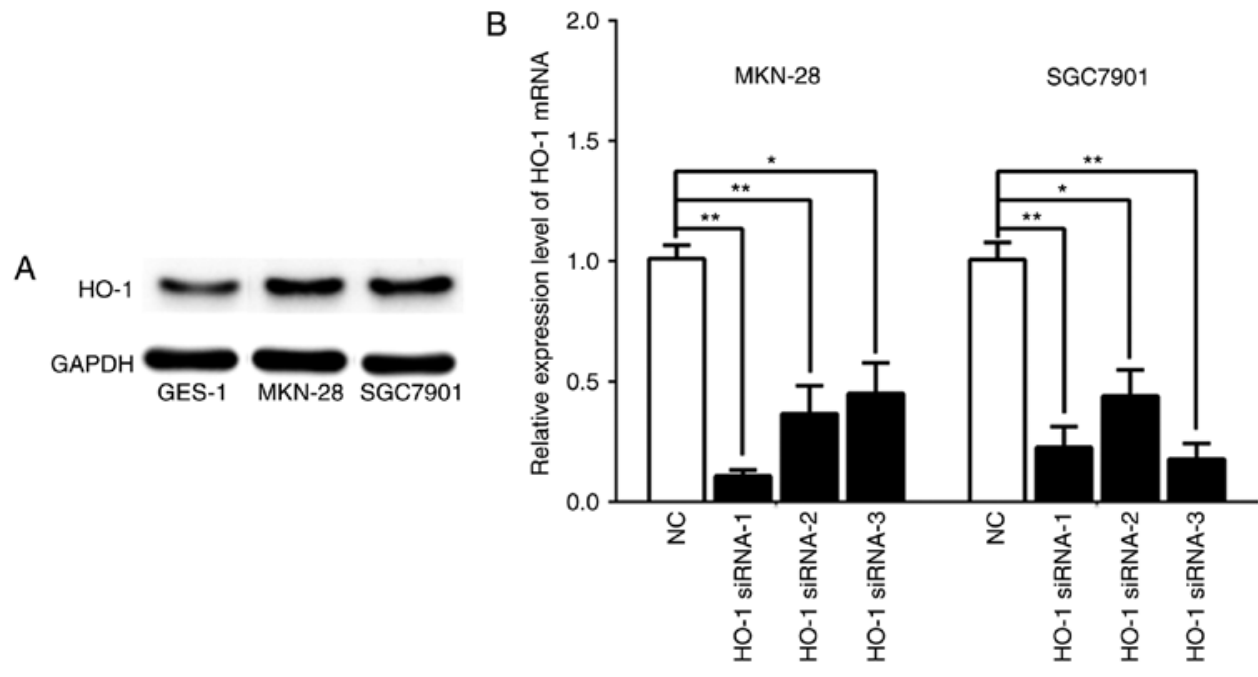

Figure 3. Protein expression of heme oxygenase-1 (HO-1) in GES-1, MKN-28, and SGC7901 cells and mRNA expression of HO-1 in MKN-28 and SGC7901 cells treated with HO-1 siRNAs. (A) The protein expression of HO-1 was higher in gastric cancer cell lines, i.e., MKN-28 and SGC7901 cells, than that in the immortal gastric epithelial cell line GES-1 (P<0.05). (B) The mRNA expression of HO-1 was significantly decreased in MKN-28 and SGC7901 cells at $24 \mathrm{~h}$ after transfection with three different strands of siRNAs when compared with the respective negative control group $\left({ }^{*} \mathrm{P}<0.05 ;{ }^{* * *} \mathrm{P}<0.01\right)$.

cance between HO-1 expression and the pathological grade of gastric cancer $(\mathrm{P}=0.052)$, and no significant correlations between HO-1 expression and the other clinicopathological characteristics, including age, sex, tumor size, clinicopathologic classifications (T, N, M), and clinical staging among the 89 gastric cancer patients $(\mathrm{P}>0.05)$.

Low expression of HO-1 is associated with an improved prognosis in gastric cancer patients. We next investigated the relationship between HO-1 expression in gastric cancer tissue and patient survival. Our results indicated that there was a significant relationship between HO-1 expression and the overall survival time in gastric cancer patients $(\mathrm{P}=0.002)$. The Kaplan-Meier curves demonstrated that the gastric cancer patients with low expression of HO-1 had a longer overall survival time (median, 33.4 months) compared with patients expressing a high level of HO-1 (median, 11.2 months, $\mathrm{P}=0.002$ ). The cumulative 5-year survival rate was $39.2 \%$ (31/79) for gastric cancer patients with low HO-1 expression. The survival rate decreased to $10 \%(1 / 10)$ for patients with high expression of HO-1 (Fig. 2).

Protein expression of HO-1 in GES-1, MKN-28, and SGC7901 cells and the effects of knockdown of $\mathrm{HO}-1$ expression on $M K N-28$ and SGC7901 cells. Western blot results indicated that the protein expression of $\mathrm{HO}-1$ was markedly higher in MKN-28 and SGC7901 cell lines compared with GES-1 cells (Fig. 3A). After confirming HO-1 expression in MKN-28 and SGC7901 cells, we downregulated the expression of HO-1 in these two cell lines using three different strands of siRNAs, namely HO-1 siRNA-1, HO-1 siRNA-2, and HO-1 siRNA-3. At $48 \mathrm{~h}$ post-transfection, the qPCR results demonstrated that the mRNA expression of HO-1 in both cell lines was significantly decreased by each strand of siRNA when compare with the respective negative control (NC) group (Fig. 3B, $\mathrm{P}<0.05$ ).

Knockdown of HO-1 expression inhibits apoptosis in gastric cancer cells. At $24 \mathrm{~h}$ post-transfection, flow cytometry analysis demonstrated that the apoptosis rates were $1.2 \pm 0.2 \%$, $10.8 \pm 1.4 \%$, and $7.5 \pm 1.9 \%$ in the MKN-28 cells transfected with NC, HO-1 siRNA-1, and HO-1 siRNA-2, respectively (Fig. 4A, upper panel). Similarly, at the same time point, the apoptosis rates were $0.4 \pm 0.2 \%, 11.8 \pm 1.1 \%$, and $8.5 \pm 1.7 \%$ in the SGC7901 cells transfected with NC, HO-1 siRNA-1, and HO-1 siRNA-2, respectively (Fig. 4A, lower panel). The knockdown of HO-1 expression by siRNAs significantly increased the apoptosis rates in MKN-28 (Fig. 4B, P<0.001) and SGC7901 cells (Fig. 4C, $\mathrm{P}<0.001$ ) when compared to their respective $\mathrm{NC}$ group.

Knockdown of HO-1 expression reduces the viability of gastric cancer cells. At 24,48 , and $72 \mathrm{~h}$ post-transfection, the MTT assay was used to measure cell viability in MKN-28 and SGC7901 cells transfected with either NC-RNA, HO-1 siRNA-1, or HO-1 siRNA-2 (Fig. 5). At $72 \mathrm{~h}$ post-transfection, the MKN-28 and SGC7901 cells transfected with HO-1 siRNAs had less viable cells compared with their respective NC group $(\mathrm{P}<0.001)$.

Knockdown of HO-1 expression attenuates invasion of gastric cancer cells. The invasive ability of MKN-28 and SGC7901 cells was evaluated at $24 \mathrm{~h}$ post-HO-1 siRNA transfection. Knockdown of HO-1 significantly attenuated cell invasion in both cell lines when compared to their respective $\mathrm{NC}$ group (Fig. 6, P<0.05).

\section{Discussion}

The expression and function of HO-1, a novel spy gene, are variable in the physiological, inflammatory, and cancerous states. HO-1 removes the toxic heme by breaking it down to biliverdin, iron ions, and carbon monoxide (9). In the physiological state, the HO-1 expression is extremely low and maintains homeostasis in the microenvironment of local tissues. Upon injury, inflammation, and other external insults in local tissues, the expression of HO-1 is elevated and serves 

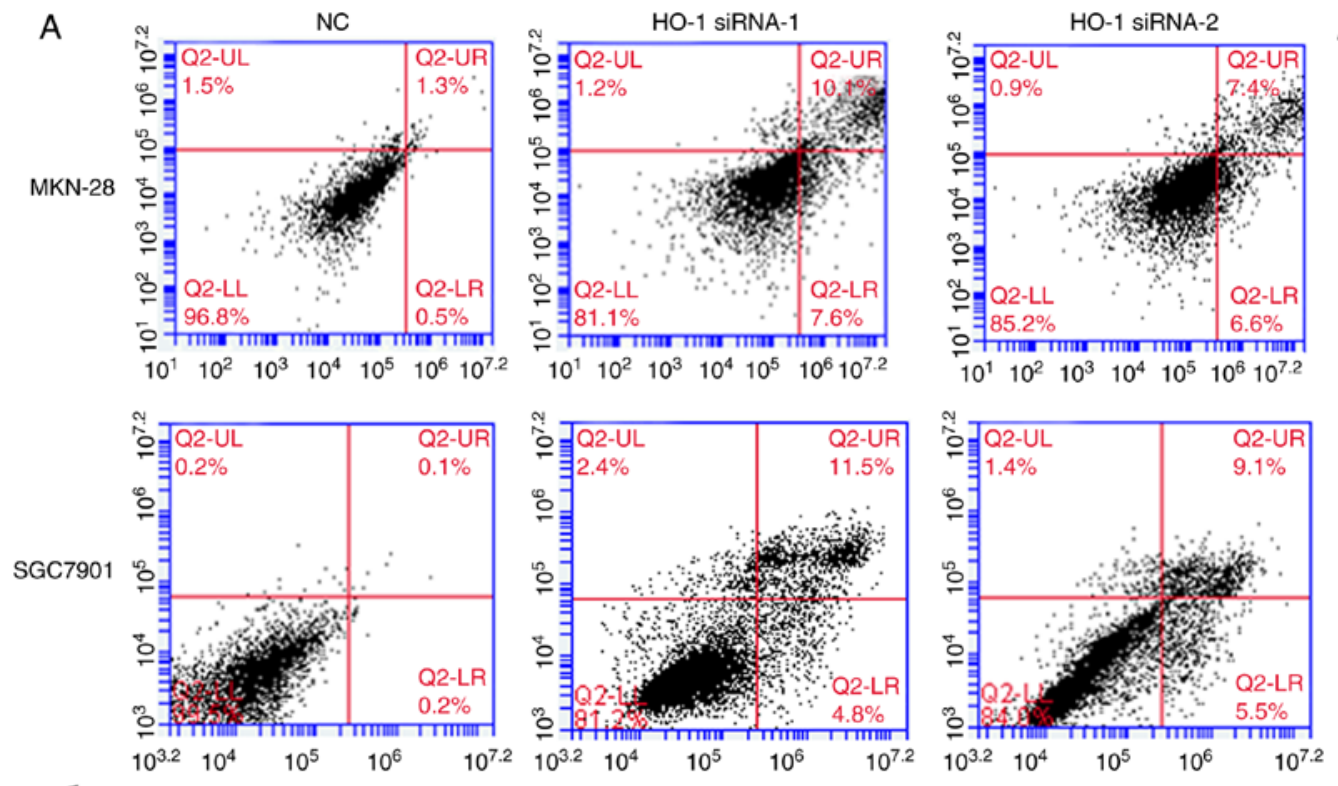

$\mathrm{PI}$

Annexin V
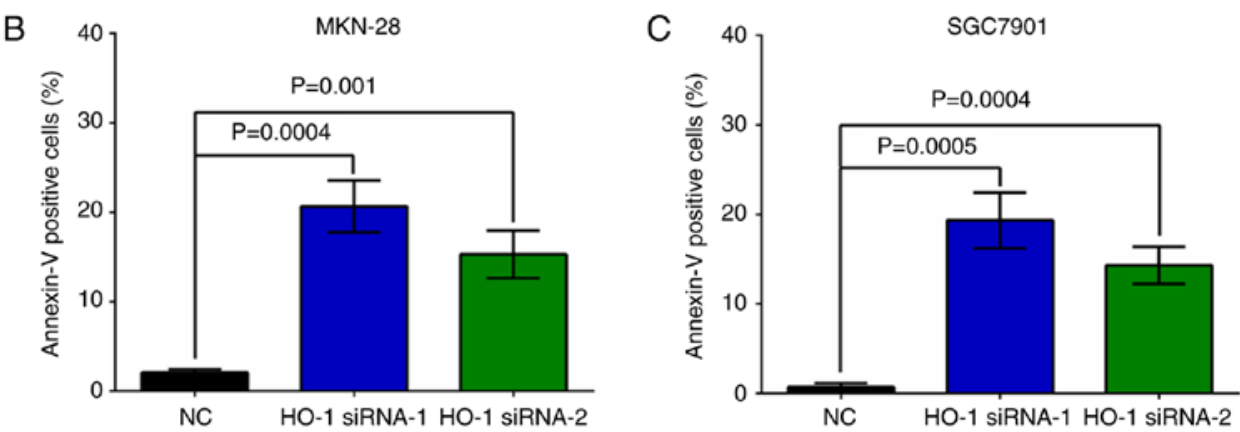

Figure 4. Knockdown of heme oxygenase-1 (HO-1) expression promotes cell apoptosis in MKN-28 and SGC7901 cells. The flow cytometry results demonstrated increased apoptosis in the cells treated with either HO-1 siRNA-1 or HO-1 siRNA-2 for $24 \mathrm{~h}$ (A). The percent Annexin V positive cells was significantly higher in HO-1 siRNA-transfected MKN-28 cells (B) and SGC7901 cells (C) when compared to their respective negative control group (P<0.05).

A

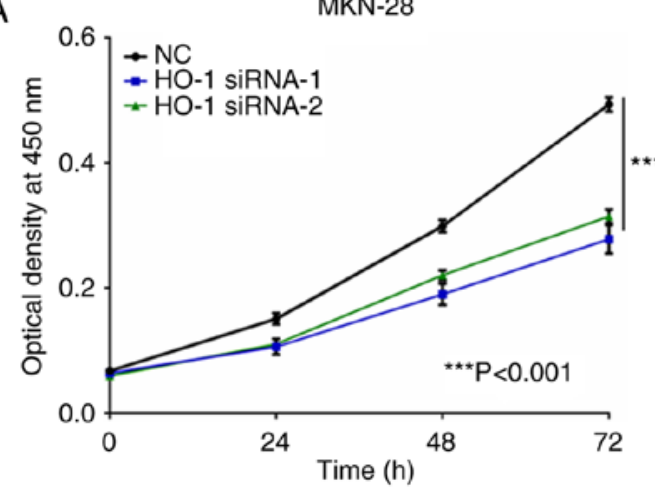

B

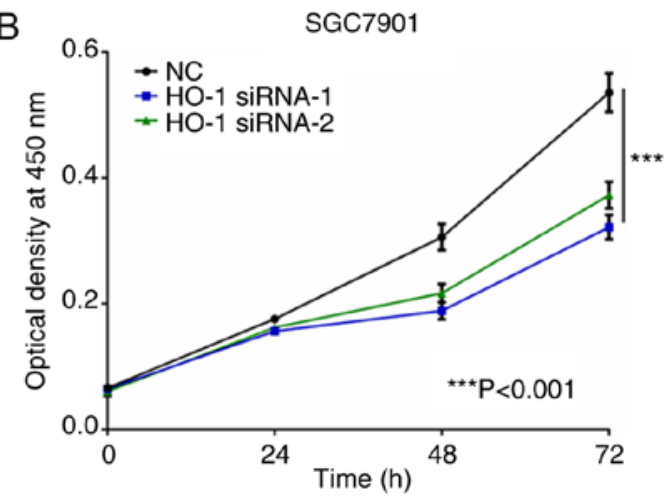

Figure 5. Knockdown of heme oxygenase-1 (HO-1) expression reduces cell viability in MKN-28 and SGC7901 cells. The MTT assay found that the cell viability was significantly decreased in the HO-1 siRNA-treated MKN-28 (A) and SGC7901 (B) cells at $72 \mathrm{~h}$ post-transfection when compared to their respective negative control group $(\mathrm{P}<0.001)$.

as a protective mechanism for the body to fight against inflammation, oxidation, and apoptosis $(10,18)$. When the harmful stimulations subside, the HO-1 expression returns to baseline (19). Chronic inflammation may lead to the development of various types of cancers, including gastric cancer (3). High expression of HO-1 has been found in various cancers, with correlations with the clinical characteristics and prognosis in patients. The prolonged high expression of HO-1 is harmful to the cancerous tissues and is associated with a poor prognosis in numerous cancers, including breast (8), colorectal (6), and prostate cancers (20). However, the role of HO-1 in gastric cancer has yet to be determined. 
A
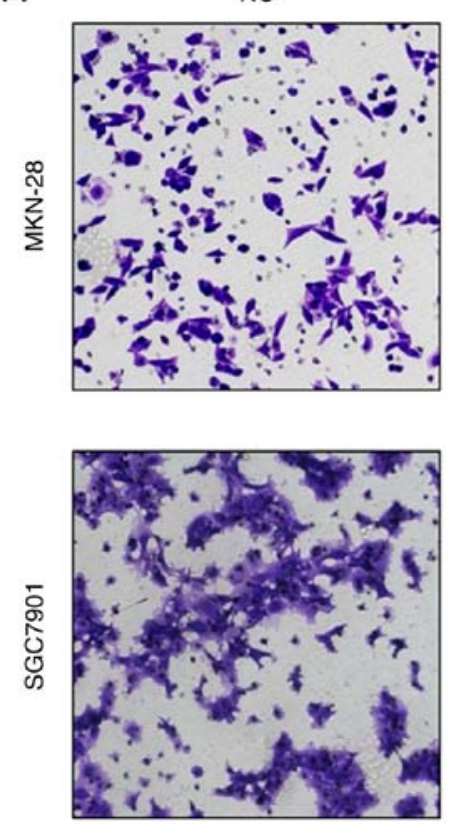

B

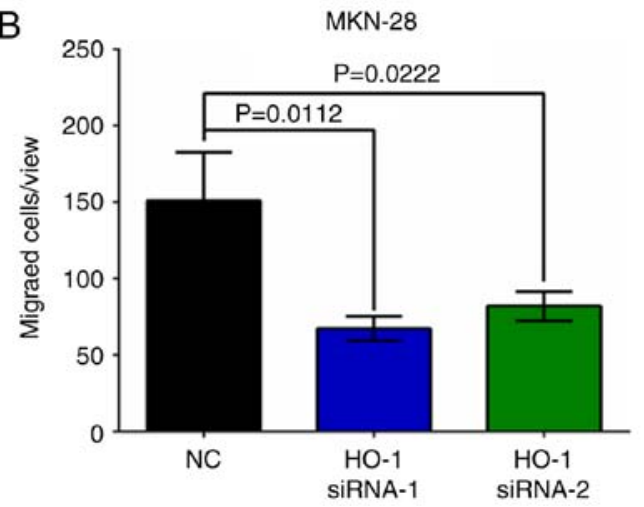

HO-1 SiRNA-1
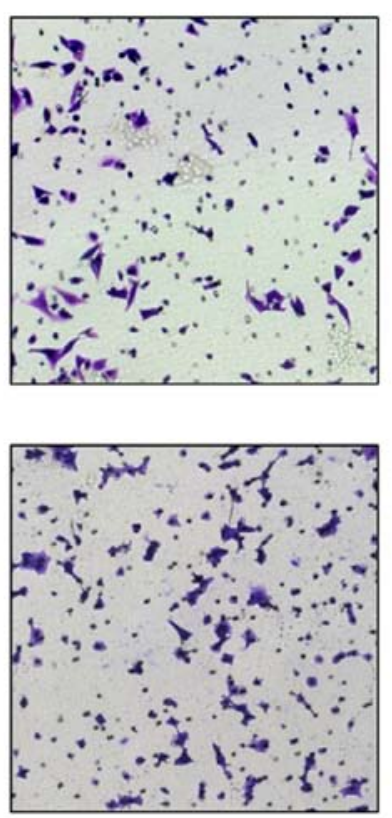

HO-1 SiRNA-2
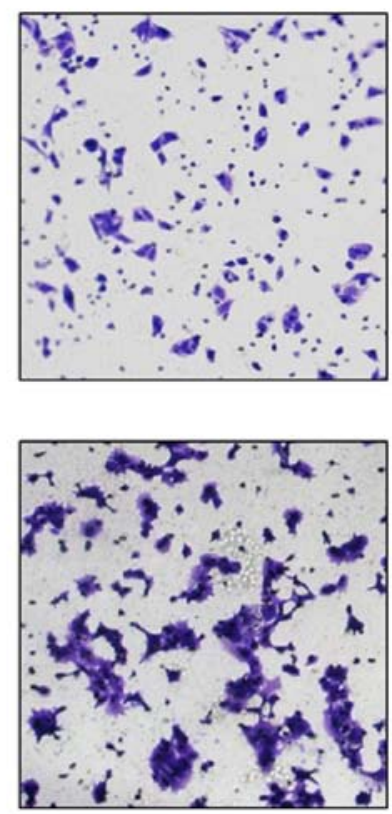

C

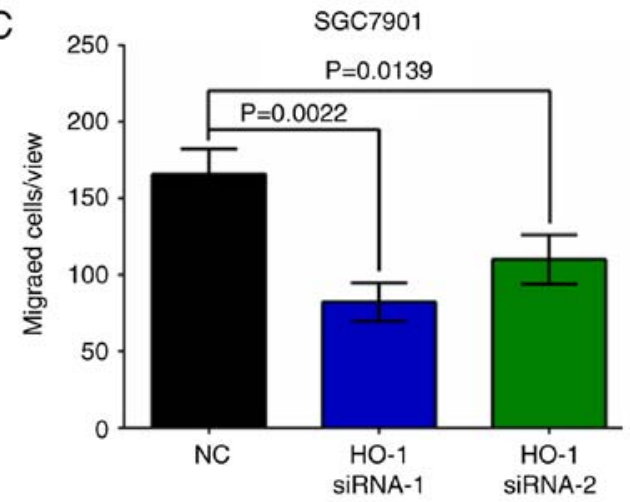

Figure 6. Knockdown of heme oxygenase-1 (HO-1) expression attenuates cell invasion in MKN-28 and SGC7901 cells. The Transwell assay found that the invasion ability was reduced in the HO-1 siRNA-treated MKN-28 (A, upper panel, and B) and SGC7901 (A, lower panel, and C) cells at 24 h post-transfection when compared to their respective negative control group $(\mathrm{P}<0.05)$.

Previous studies demonstrated that upregulation of HO-1 leads to resistance of apoptosis in gastric cancer cells (21). In addition, the DNA polymorphism in the HO-1 promoter is associated with the risk of developing gastric adenocarcinoma (22). Expression of HO-1 was detected in 62 gastric cancer tissues of 74 cases (83.8\%) (23). This evidence suggests that HO-1 may play a vital role in gastric carcinogenesis. At present, scarce data have shown whether the expression of HO-1 is higher in gastric cancer compared to matched adjacent non-cancer tissues or investigated the relationship between HO-1 expression and overall survival of gastric cancer patients.

Our present study found that $11 \%$ gastric cancer tissues had high expression of HO-1 compared to only $1 \%$ matched adjacent normal tissues had HO-1 expression. We are the first to demonstrate that low HO-1 expression in gastric cancer patients was positively correlated with their overall survival. This result is consistent with findings from previous studies that found HO-1 expression is elevated in breast cancer tissues and associated with the prognosis of breast cancer patients (8). However, our analysis did not find correlations between HO-1 expression with either the pathological grade or clinical characteristics of gastric cancer. This result differs from previous studies and may be due to a limited number of cases or demographical reasons.

Several studies have reported that low expression of HO-1 promotes apoptosis in acute myeloid leukemia cells and that increased HO-1 expression inhibits the chemotherapy-induced apoptosis $(24,25)$. HO-1 influences apoptosis in human acute myeloid leukemia by regulating the transcription factors $\mathrm{Nrf} 2$, $\mathrm{NF}-\kappa \mathrm{B}$, and AP-1. In contrast, knockdown of HO-1 expression in gastric cancer cells by siRNA sensitizes the cells to chemotherapy (23). In this study, we found that the two gastric cancer lines, MKN-28 and SGC7901 cells, had higher protein expression of HO-1 compared with the immortalized normal gastric epithelial cell line GES-1. For more reliable results, we used two strands of siRNAs targeting HO-1 to independently knock down HO-1 expression in MKN-28 and SGC7901 cells. Knockdown of HO-1 significantly increased the apoptosis rate in gastric cancer cells, which is consistent with previous studies.

An increasing number of studies have identified the correlation between HO-1 expression and cancer cell proliferation. 
For example, cell proliferation is inhibited in HO-1 siRNAtreated human pancreatic cancer cells and bladder cancer cell lines $(26,27)$. In addition, downregulation of HO-1 in human urothelial cancer cell lines decreases the expression of Ki-67, a marker for cell proliferation (28). Our data are consistent with these findings. In gastric cancer cell lines, the proliferation was significantly decreased at $72 \mathrm{~h}$ after HO-1 siRNA transfection. To our knowledge, little is known about how HO-1 regulates cell proliferation so far, and more research is needed to investigate this mechanism.

It has been reported that increased expression of HO-1 promotes the invasive ability of lung adenocarcinoma cell lines, while downregulation of HO-1 expression by siRNA inhibits their invasive ability $(29,30)$. ROS and the TGF- $\beta 1 / P I 3 K / A k t$ signaling pathway may be the mechanism involved in HO-1 regulation of cell invasion. In another study, upregulation of HO-1 expression in gastric cancer cells increased the expression of MMP, a biomarker for cell invasion and migration, and vice versa (23). In this study, we found that knockdown of HO-1 expression attenuated gastric cancer cell invasion, strongly agreeing with the previous studies.

In conclusion, our results demonstrated that low expression of HO-1 in gastric cancer tissues correlated with a better prognosis in patients and that knockdown of the HO-1 expression inhibited gastric cancer cell apoptosis, proliferation, and invasion. More research is needed to elucidate the molecular mechanisms, but our findings suggest that the HO-1 gene can be targeted to treat gastric cancer.

\section{References}

1. Torre LA, Bray F, Siegel RL, Ferlay J, Lortet-Tieulent J and Jemal A: Global cancer statistics, 2012. CA Cancer J Clin 65: 87-108, 2015

2. de Korwin J-D: Epidemiology of Helicobacter pylori infection and gastric cancer. Rev Prat 64: 189-193, 2014 (In French).

3. Munn LL: Cancer and inflammation. Wiley Interdiscip Rev Syst Biol Med 9: e1370, 2017.

4. Vohlonen I, Pukkala E, Malila N, Härkönen M, Hakama M, Koistinen V and Sipponen P: Risk of gastric cancer in Helicobacter pylori infection in a 15-year follow-up. Scand J Gastroenterol 51: $1159-1164,2016$

5. Jozkowicz A, Was H and Dulak J: Heme oxygenase-1 in tumors: Is it a false friend? Antioxid Redox Signal 9: 2099-2117, 2007.

6. Yin H, Fang J, Liao L, Maeda H and Su Q: Upregulation of heme oxygenase-1 in colorectal cancer patients with increased circulation carbon monoxide levels, potentially affects chemotherapeutic sensitivity. BMC Cancer 14: 436, 2014.

7. Degese MS, Mendizabal JE, GandiniNA, Gutkind JS, Molinolo A, Hewitt SM, Curino AC, Coso OA and Facchinetti MM: Expression of heme oxygenase-1 in non-small cell lung cancer (NSCLC) and its correlation with clinical data. Lung Cancer 77: $168-175,2012$

8. Noh SJ, Bae JS, Jamiyandorj U, Park HS, Kwon KS, Jung SH, Youn HJ, Lee H, Park BH, Chung MJ, et al: Expression of nerve growth factor and heme oxygenase-1 predict poor survival of breast carcinoma patients. BMC Cancer 13: 516, 2013.

9. Wegiel B, Nemeth Z, Correa-Costa M, Bulmer AC and Otterbein LE: Heme oxygenase-1: A metabolic nike. Antioxid Redox Signal 20: 1709-1722, 2014.

10. Lundvig DM, Immenschuh S and Wagener FA: Heme oxygenase, inflammation, and fibrosis: The good, the bad, and the ugly? Front Pharmacol 3: 81, 2012.

11. Ivanov AV, Valuev-Elliston VT, Tyurina DA, Ivanova ON, Kochetkov SN, Bartosch B and Isaguliants MG: Oxidative stress, a trigger of hepatitis $C$ and $B$ virus-induced liver carcinogenesis. Oncotarget 8: 3895-3932, 2017.
12. Liao YF, Zhu W, Li DP and Zhu X: Heme oxygenase-1 and gut ischemia/reperfusion injury: A short review. World $J$ Gastroenterol 19: 3555-3561, 2013.

13. Strasser-Weippl K and Ludwig H: Ferritin as prognostic marker in multiple myeloma patients undergoing autologous transplantation. Leuk Lymphoma 55: 2520-2524, 2014.

14. Torti SV and Torti FM: Iron and cancer: More ore to be mined. Nat Rev Cancer 13: 342-355, 2013.

15. Zheng J, Nagda DA, Lajud SA, Kumar S, Mouchli A, Bezpalko O, O'Malley BW Jr and Li D: Biliverdin's regulation of reactive oxygen species signalling leads to potent inhibition of proliferative and angiogenic pathways in head and neck cancer. Br J Cancer 110: 2116-2122, 2014

16. Yang SL, Liu LP, Jiang JX, Xiong ZF, He QJ and Wu C: The correlation of expression levels of HIF-1 $\alpha$ and HIF-2 $\alpha$ in hepatocellular carcinoma with capsular invasion, portal vein tumor thrombi and patients' clinical outcome. Jpn J Clin Oncol 44: 159-167, 2014.

17. Hu JL, Xiao L, Li ZY, Wang Q, Chang Y and Jin Y: Upregulation of HO-1 is accompanied by activation of p38MAPK and mTOR in human oesophageal squamous carcinoma cells. Cell Biol Int 37: 584-592, 2013.

18. Furfaro AL, Traverso N, Domenicotti C, Piras S, Moretta L, Marinari UM, Pronzato MA and Nitti M: The Nrf2/HO-1 axis in cancer cell growth and chemoresistance. Oxid Med Cell Longev 2016: 1958174, 2016.

19. Trachootham D, Alexandre J and Huang P: Targeting cancer cells by ROS-mediated mechanisms: A radical therapeutic approach? Nat Rev Drug Discov 8: 579-591, 2009.

20. Alaoui-Jamali MA, Gupta A, Szarek WA, Bismar TA, Gheorghe R and Schipper HM: A novel selective therapeutic targeting heme oxygenase- 1 revealed a potent antimetastatic activity in androgen-refractory human prostate cancer models. J Clin Oncol 27: e16090, 2009. doi: 10.1200/jco.2009.27.15s.e16090.

21. Liu ZM, Chen GG, Ng EKW, Leung WK, Sung JJY and Chung SCS: Upregulation of heme oxygenase-1 and p21 confers resistance to apoptosis in human gastric cancer cells. Oncogene 23: 503-513, 2004

22. Motovali-Bashi $\mathbf{M}$ and Hamidy $M$ : Association between GT-repeat polymorphism at heme oxygenase-1 gene promoter and gastric cancer and metastasis. Tumour Biol 36: 4757-4762, 2015.

23. Yin Y, Liu Q, Wang B, Chen G, Xu L and Zhou H: Expression and function of heme oxygenase-1 in human gastric cancer. Exp Biol Med (Maywood) 237: 362-371, 2012.

24. Mayerhofer M, Florian S, Krauth MT, Aichberger KJ Bilban M, Marculescu R, Printz D, Fritsch G, Wagner O, Selzer E, et al: Identification of heme oxygenase-1 as a novel BCR/ABL-dependent survival factor in chronic myeloid leukemia. Cancer Res 64: 3148-3154, 2004.

25. Heasman SA, Zaitseva L, Bowles KM, Rushworth SA and Macewan DJ: Protection of acute myeloid leukaemia cells from apoptosis induced by front-line chemotherapeutics is mediated by haem oxygenase-1. Oncotarget 2: 658-668, 2011.

26. Berberat PO, Dambrauskas Z, Gulbinas A, Giese T, Giese N, Künzli B, Autschbach F, Meuer S, Büchler MW and Friess H: Inhibition of heme oxygenase-1 increases responsiveness of pancreatic cancer cells to anticancer treatment. Clin Cancer Res 11: 3790-3798, 2005

27. Miyake M, Ishii M, Kawashima K, Kodama T, Sugano K, Fujimoto K and Hirao Y: siRNA-mediated knockdown of the heme synthesis and degradation pathways: Modulation of treatment effect of 5-aminolevulinic acid-based photodynamic therapy in urothelial cancer cell lines. Photochem Photobiol 85: 1020-1027, 2009.

28. Miyake M, Fujimoto K, Anai S, Ohnishi S, Nakai Y, Inoue T, Matsumura Y, Tomioka A, Ikeda T, Okajima E, et al: Inhibition of heme oxygenase- 1 enhances the cytotoxic effect of gemcitabine in urothelial cancer cells. Anticancer Res 30: 2145-2152, 2010.

29. Jeon WK, Hong HY, Seo WC, Lim KH, Lee HY, Kim WJ, Song SY and Kim BC: Smad7 sensitizes A549 lung cancer cells to cisplatin-induced apoptosis through heme oxygenase-1 inhibition. Biochem Biophys Res Commun 420: 288-292, 2012.

30. Zhang W, Qiao T and Zha L: Inhibition of heme oxygenase-1 enhances the radiosensitivity in human nonsmall cell lung cancer a549 cells. Cancer Biother Radiopharm 26: 639-645, 2011. 